\title{
Evaluation and control of waste anesthetic gas in the postanesthesia care unit within patient and caregiver breathing zones
}

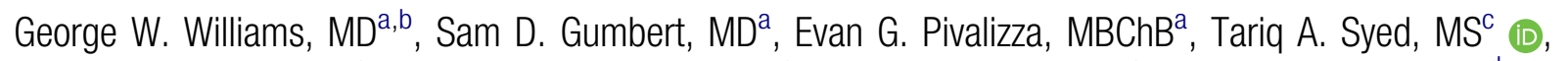
Tyrone Burnett, Jr., BS ${ }^{\mathrm{C}}$ (D), Omar L. Mancillas, $\mathrm{MD}^{\mathrm{c}}$, Leslie A. Vargas, MDª, Stephanie H. Ahn, BS ${ }^{\mathrm{d}} \mathbb{1}$, Chunyan Cai, $\mathrm{PhD}^{\mathrm{e}}$, (D) and Carin A. Hagberg, $\mathrm{MD}^{\mathrm{C}}$

${ }^{a}$ Department of Anesthesiology, The University of Texas Health Science Center at Houston, McGovern Medical School, Houston, Texas; ${ }^{b}$ Department of Neurosurgery, The University of Texas Health Science Center at Houston, McGovern Medical School, Houston, Texas; 'Department of Anesthesiology, Critical Care, and Pain Medicine, The University of Texas MD Anderson Cancer Center, Houston, Texas; 'Department of Surgery, The University of Texas Health Science Center at Houston, McGovern Medical School, Houston, Texas;

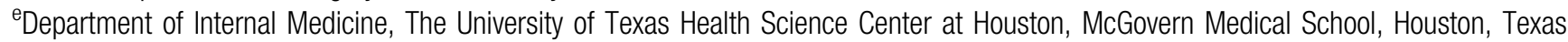

\begin{abstract}
This study (NCT02428413) evaluated waste anesthetic gas (WAG) in the postanesthesia care unit (PACU) and assessed the utility of the ISO-Gard ${ }^{\circledR}$ mask in reducing nursing exposure to WAG. We hypothesized that WAG levels in the patient's breathing zone upon recovery would exceed the recommended levels, leading to increased exposure of the PACU nurses, with use of the ISOGard mask limiting this exposure. A total of 125 adult patients were recruited to participate. Patients were randomized to receive the standard oxygen delivery mask or the ISO-Gard face mask postoperatively. Continuous particulate concentrations were measured using infrared spectrophotometers placed within the patients' and nurses' 6-inch breathing zone. Maximum WAG measurements were obtained every 30 seconds, and the duration of maximum WAG $>2 \mathrm{ppm}$ and its proportion relative to the total collection period were calculated. We observed a statistically significant difference in desflurane duration and proportion of maximum WAG $>2$ ppm in both patient and PACU nurse breathing zones. Therefore, patients and PACU nurses using routine care were exposed to WAG levels $>2 \mathrm{ppm}$ during the 1-hour postoperative period, and the ISO-Gard mask effectively reduced the amount of WAG detected in the immediate 1 -hour postoperative recovery phase.
\end{abstract}

KEYWORDS Anesthetic pharmacokinetics; occupational safety; waste anesthetic gas

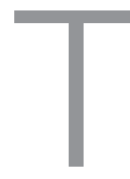

he scavenging of waste anesthetic gases (WAG) is recommended by the Occupational Safety and Health Administration in environments that use anesthetic gases to reduce occupational exposure. ${ }^{1}$ Exposure is determined by the concentration of agent in the breathing zone, which the Occupational Safety and Health Administration defines as an area surrounding the face encompassing approximately 6 to 9 inches, and the length of time that the agent is continuously inhaled. ${ }^{2,3}$ Recommended levels apply anywhere that anesthetic agents are delivered, as well as to the postanesthesia care unit
(PACU). ${ }^{1}$ Though random room samples may indicate low levels of WAG, the breathing zone of nurses near a recovering patient may expose them to levels of anesthetic gases that are above the National Institute of Occupational Safety and Health's (NIOSH) recommendation of concentrations $>2$ ppm over a sampling period not to exceed 1 hour. ${ }^{4}$ Recent studies suggest that potential PACU nurse exposure to WAG is above NIOSH guidelines, given that such levels in the patient breathing zone exceed these guidelines and may occur as much as $49 \%$ of the time. ${ }^{5,6}$ With this in mind, exposure has been associated with multiple medical

Corresponding author: George W. Williams II, MD, FASA, FCCP, Departments of Anesthesiology and Neurosurgery, The University of Texas Health Science Center at Houston, McGovern Medical School, 6431 Fannin, MSB 5.020, Houston, TX 77030-1501 (email: george.w.willams@uth.tmc.edu)

Color versions of one or more of the figures in the article can be found online at www.tandfonline.com/ubmc.

Received April 10, 2018; Revised July 10, 2018; Accepted July 16, 2018. 
problems including headaches, irritability, fatigue, nausea, drowsiness, and difficulty with judgment and coordination. ${ }^{3}$ Though operating rooms have implemented techniques to decrease occupational exposure by scavenging WAG, thus minimizing these potential health consequences, few studies address the risk of PACU WAG exposure.

\section{METHODS}

The purpose of this observational prospective study was to evaluate the use of the ISO-Gard ${ }^{\circledR}$ mask (Figure 1a), a 510(k) approved class II/nonsignificant risk device (cleared by the US Food and Drug Administration). The study had three primary objectives: (1) measure the level of WAG emanating from the patient during the immediate 1-hour postoperative recovery period in the PACU; (2) measure the level of WAG within the PACU nurse's breathing zone during this immediate 1-hour postoperative recovery period; and (3) validate the efficacy of the ISO-Gard mask in reducing nurse exposure to WAG in the PACU.

The ISO-Gard ClearAir ${ }^{\text {TM }}$ system (Teleflex Inc., Morrisville, NC) is a modified oxygen delivery face mask that actively scavenges WAG exhaled by patients recovering from surgery in the PACU, allowing for the delivery of supplemental and/or therapeutic oxygen to patients to aid in their recovery while simultaneously reducing the amount of expelled WAG released to the work environment. Suction for the scavenging of WAG through the attached corrugated hose is provided by the institution's regulated vacuum source. Excess anesthetic gases can be removed with the use of negative pressure through the facility's central vacuum. The interface of the machine is connected to the gas disposal system of the facility, which vents the waste gases outside the
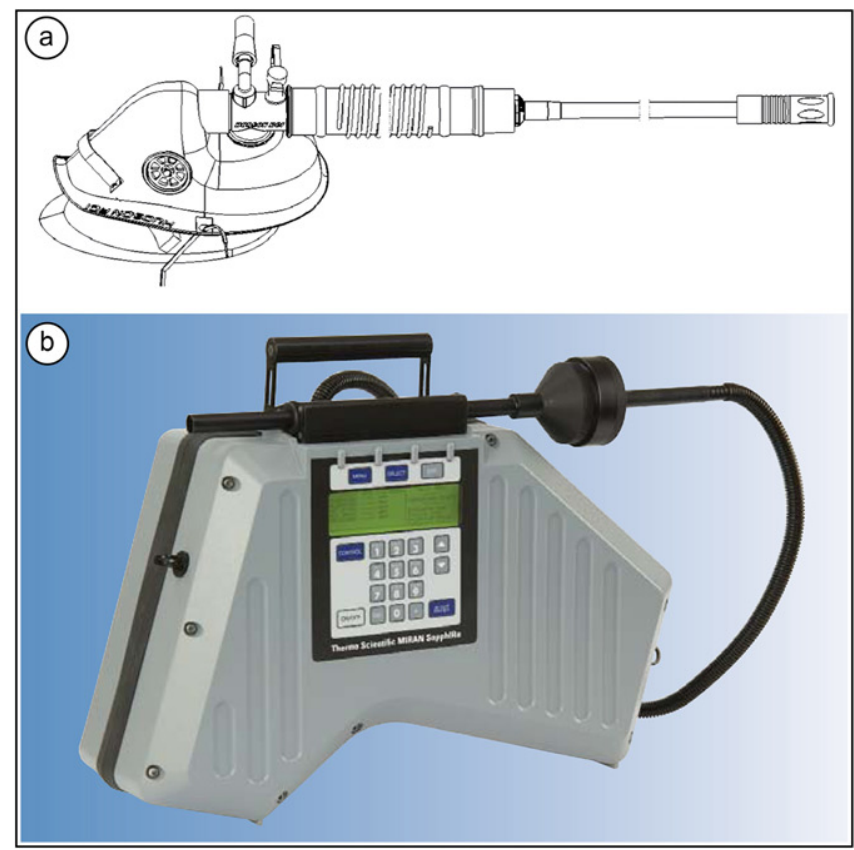

Figure 1. (a) ISO-Gard mask. (b) The MIRAN 1B SapphIRe Ambient Air Analyzer. building away from any return ducts or open windows. The mask can be used with or without the vacuum to function as a standard oxygen delivery mask with an end-tidal carbon dioxide monitoring port. The user may adjust the oxygen flow rate and the vacuum rate as needed to deliver the required oxygen and effectively scavenge the WAG.

Following institutional review board approval, informed consent was obtained preoperatively from patients by key study personnel. Once informed consent had been obtained, the patient's medical history and physical exam as documented by the anesthesiologist was reviewed, as well as the planned anesthetic technique and estimated operative time, to confirm that the inclusion/exclusion criteria were met and the patient was eligible for participation.

Eligible patients met all of the following inclusion criteria: (1) age $\geq 18$ years, (2) expected surgery duration $\geq 2$ hours, (3) requirement of inhalation halogenated anesthetic gas (i.e., sevoflurane or desflurane) as the primary modality of anesthesia during surgery, (4) life expectancy of $>1$ year (not documented as terminally ill, American Society of Anesthesiologists classification I to III), and (5) expected stay in the PACU of at least 1 hour following surgery.

The following criteria were used for exclusion: (1) requirement for $>23$-hour hospital stay (or inpatient surgery), (2) planned use of total intravenous anesthesia, (3) inability to tolerate the use of a mask in the PACU, (4) spinal or neurosurgery, (5) previous study enrollment, (6) employment with the investigator or involvement in other studies under the direction of the investigator or study site, (7) pregnancy, and (8) $>45$ minutes between the end of anesthetic gas administration and arrival in the PACU.

A total of 125 adult patients, scheduled for an outpatient elective surgical procedure at Memorial Hermann Hospital-Texas Medical Center, were enrolled in the study. Inclusion criteria were validated, and patients were randomized to the traditional device group (control mask) or the ISO-Gard mask group (study mask) postoperatively. Within each device group were two population subsets, patients who received sevoflurane or desflurane as the primary anesthetic modality.

All elements of patient care followed the institution's standard of practice. The patients underwent general anesthesia as prescribed by the attending anesthesiologist. If it was determined perioperatively that a multimodal anesthetic was necessary, the patient was excluded from the study. If anesthetic gas was the primary modality utilized, then the patient was monitored according to the study protocol.

In the operating room, standard American Society of Anesthesiologists monitors were applied. General anesthesia was induced by bolus administration of propofol $(1.5$ to $2 \mathrm{mg} / \mathrm{kg})$ and fentanyl $(1 \mathrm{mcg} / \mathrm{kg})$, and rocuronium $(0.6 \mathrm{mg} / \mathrm{kg})$ was administered to provide muscle relaxation. The patient's lungs were ventilated via anesthesia face mask and $100 \%$ oxygen until the patient was completely relaxed (train of four on twitch monitor was 0 ). The patient's airway 
was then secured either by placement of a supraglottic airway or laryngoscopy and endotracheal intubation. Anesthesia was maintained with either sevoflurane or desflurane inhalational agents. The lungs were mechanically ventilated with a semiclosed circle system to maintain an end-tidal carbon dioxide near $35 \mathrm{~mm} \mathrm{Hg}$.

Following the operative case, patients were transported to the PACU based on standard procedure and their arrival time was documented. The monitoring equipment was set up for the patient and for the PACU nurse assigned prior to PACU arrival. Patient "hook-up" to the assigned device attached to the wall was recorded as zero time, and recording of WAG was initiated. The ISO-Gard mask was used as directed by the manufacturer (oxygen flow rate of $6 \mathrm{~L} / \mathrm{min}$ and suction flow rate of $30 \mathrm{~mm} \mathrm{Hg}$ ). WAG concentrations were measured with the MIRAN 1B Analyzer wand supported by a microphone stand within the patient's breathing zone for 60 minutes. WAG concentrations were measured with the MIRAN 1B Analyzer with the sampling point affixed to the caregiver's shoulder within his or her breathing zone for 60 minutes. Each MIRAN 1B Analyzer was calibrated daily prior to use and zeroed before each data collection to prevent zero drift error. Sevoflurane or desflurane WAG environmental air concentrations were measured continuously at 30-second intervals, in parts per million resolutions, using infrared spectrophotometry.

A research assistant was present throughout the duration of the patient's PACU stay to troubleshoot and monitor complications with the mask or the MIRAN 1B Analyzer (Figure 1b) or to assist the nurse, if needed. The length of time the mask was used was observed with the "mask off time" recorded. At the end of the 1-hour measuring period, the equipment was collected and the patients were discharged from the PACU via standard procedure.

There was no notable risk to participants of this study. In the control arm (standard face mask), study personnel only monitored WAG levels in the PACU under normal working conditions. The test arm utilized the ISO-Gard mask to deliver supplemental oxygen to patients while simultaneously scavenging WAG. The risks associated with its use are similar to the risks associated with using standard supplemental oxygen masks. There are no known effects or dangers to patients undergoing breathing zone analysis, and this technique has been used safely in a previous pilot study. ${ }^{6}$

It was the goal of this study to determine not only the amount of WAG outgassed from the patient postoperatively but also the amount of nurse exposure. To do this, all PACU nurses were approached for participation because they had the potential to care for study patients in the PACU during the 1 -hour monitoring period. During the study, the nurses were required to wear a gas sampling line. Informed consent was obtained from all PACU nurses to allow on-person monitoring for WAG with a sampling point affixed to their shoulder, representing the reach of their breathing zone. Nurses assigned to patients randomized to the ISO-
Gard mask were also required to answer a survey after patient discharge from the PACU pertaining to their experience with the mask, and all responses were noted. The nurses worked according to their standard of practice and were permitted to move about freely within a 5-m radius (hose length).

Study personnel and participating nurses were oriented to and instructed on use of the scavenging mask according to product labeling. The research team was trained on study execution, data collection, use of the MIRAN 1B Analyzer, and reporting procedures specific to the study protocol. All training was conducted under good clinical practice prior to patient enrollment, and periodic monitoring visits were made to ensure protocol adherence.

A sample size of 100 patients-that is, 50 patients per group-was able to detect an effect size of 0.57 at $80 \%$ power, $95 \%$ confidence interval, and level of significance of $P=0.05$. Patients were allocated according to a randomized block scheme with a block size of four in a 1:1 allocation ratio.

Demographics, vital signs, laboratory variables, adverse events/serious adverse events, and WAG levels were summarized for each group. The distribution of all variables was examined to check the validity of distribution assumptions before any analysis, using measures of central tendency and a visual inspection of histograms and quantile-quantile plots. Where possible, those not meeting normality assumptions were transformed to approximate a normal distribution, to allow the use of parametric tests. Where data could not be transformed, equivalent nonparametric tests were employed. Descriptive statistics (mean $\pm S D$ for continuous variables and frequency [percentage] for discrete variables) were summarized for all variables. Continuous variables with a normal distribution were reported as mean and SD and continuous variables with skewed distribution were summarized as median and interquartile ranges. Continuous variables were analyzed by linear regression with $P$ values obtained by logistic regression. Categorical variables were reported as frequency and percentage using a two-sample Student's $t$ test for statistical analysis with $P$ values obtained by chisquare test.

The maximum, minimum, average, and cumulative WAG levels in the PACU between study and control groups were compared by two-sample $t$ test or Wilcoxon rank sum test, as appropriate. The percentage of patients in which the average WAG exposure exceeded recommended levels was calculated and compared by chi-square test or Fisher's exact test, as appropriate. We also calculated and compared the duration of the maximum (MAX) WAG levels exceeding the recommended levels between the two groups.

For MAX-WAG measurements obtained every 30 seconds, we calculated the duration of MAX-WAG $(>2 \mathrm{ppm})$ and its proportion relative to the total collection period. Wilcoxon's rank sum test was applied to conduct the comparison on the duration with MAX-WAG ( $>2 \mathrm{ppm}$ ) and its proportion between two groups. All statistical 
analyses were performed using SAS 9.4 (SAS Institute Inc., Cary, NC).

\section{RESULTS}

One hundred twenty-five patients in this study received either sevoflurane or desflurane inhaled anesthetic gas. No adverse events related to the ISO-Gard mask were noted. Equipment failure led to the exclusion of 17 cases from the data analyses. Of the 108 included cases, women made up $64 \%$ of the participants (Table 1).

Twenty-four PACU nurses participated in this study over the course of 4 months. They were surveyed at the conclusion of each case, with most reporting "good" to "excellent"

Table 1. Study population

\begin{tabular}{|c|c|c|c|c|c|c|}
\hline & \multicolumn{3}{|c|}{ All cases } & \multicolumn{3}{|c|}{ Included cases } \\
\hline & Total & Female & Male & Total & Female & Male \\
\hline Total (\%) & 125 & 79 (63.2\%) & $46(36.8 \%)$ & 108 & 69 (63.9\%) & 39 (36.1\%) \\
\hline Control cases & 64 & 44 & 20 & 56 & 41 & 15 \\
\hline Desflurane & 35 & 30 & 5 & 32 & 27 & 5 \\
\hline Sevoflurane & 29 & 14 & 15 & 24 & 14 & 10 \\
\hline Iso-Gard cases & 61 & 35 & 26 & 52 & 28 & 24 \\
\hline Desflurane & 33 & 21 & 12 & 31 & 19 & 12 \\
\hline Sevoflurane & 28 & 14 & 14 & 21 & 9 & 12 \\
\hline
\end{tabular}

integration of the device into standard procedures with minimal impedance to workflow.

There was no significant difference in randomization between study and control groups. Fifty-six patients made up the traditional mask group (group 0), with $57.1 \%$ in the desflurane subgroup and $42.9 \%$ in the sevoflurane group. Fiftytwo patients were in the ISO-Gard group (group 1), with $59.6 \%$ in the desflurane subgroup and $40.4 \%$ in the sevoflurane group. No significant differences were observed in duration of data collection in patients who received either inhaled gas in either mask group.

As shown in Table 2, within the patient's breathing zone, the median duration of MAX-WAG ( $>2$ ppm) was 19.5 minutes in group 0 and 13.5 minutes in group 1 $(P=0.02)$. The median proportion of MAX-WAG ( $>2 \mathrm{ppm}$ ), which refers to the percentage of MAX-WAG in relation to the total collection period, within the patient's breathing zone was $32.2 \%$ in group 0 and $22.4 \%$ in group 1 $(P=0.03)$. The duration and proportion values remained significant for the subgroup of patients receiving anesthesia with desflurane ( $P=0.03$ and 0.04 , respectively) but not for the subgroup of patients receiving anesthesia with sevoflurane $(P=0.27$ and 0.33 , respectively) (Table 2$)$.

Within the nurse's breathing zone (Table 3 ), the median duration of MAX-WAG ( $>2 \mathrm{ppm}$ ) was 3.0 minutes in group 0 and 1.0 minutes in group $1(P<0.01)$. The median proportion of MAX-WAG ( $>2 \mathrm{ppm})$ was $4.7 \%$ in group 0 and $2.0 \%$ in group $1(P<0.01)$. As with the patient's breathing zone, the duration and proportion values remained significant for the subgroup of patients receiving anesthesia with desflurane but not for those receiving anesthesia with sevoflurane.

Table 2. Maximum waste anesthetic gas within the patient's breathing zone

\begin{tabular}{|c|c|c|c|}
\hline \multirow[b]{2}{*}{ Variable } & \multicolumn{2}{|c|}{ Randomization group } & \multirow[b]{2}{*}{$P$ value } \\
\hline & Traditional mask (control) & ISO-Gard mask & \\
\hline All patients (n) & 56 & 52 & \\
\hline Collection duration (min), mean \pm SD & $58.7 \pm 8.1$ & $56.7 \pm 10.2$ & $0.26^{*}$ \\
\hline Duration of MAX-WAG > 2 ppm (min), median (Q1, Q3) & $19.5(12.0,37.6)$ & $13.5(3.2,24.0)$ & 0.02 \\
\hline Proportion of MAX-WAG > 2 ppm during collection (\%), median (Q1, Q3) & $32.2 \%(20.4,61.1)$ & $22.4 \%(6.4,40.4)$ & 0.03 \\
\hline Anesthesia with desflurane (n) & 32 & 31 & \\
\hline Collection duration (min), mean \pm SD & $59.6 \pm 7.6$ & $58.4 \pm 9.0$ & $0.57^{*}$ \\
\hline Duration of MAX-WAG > 2 ppm (min), median (Q1, Q3) & $21.0(13.5,43.2)$ & $14.7(2.5,26.3)$ & 0.03 \\
\hline Proportion of MAX-WAG > 2 ppm during collection (\%), median (Q1, Q3) & $33.7 \%(22.8,75.6)$ & $23.6 \%(4.2,41.0)$ & 0.04 \\
\hline Anesthesia with sevoflurane (n) & 24 & 21 & \\
\hline Collection duration (min), mean \pm SD & $57.4 \pm 8.7$ & $54.1 \pm 11.6$ & $0.28 *$ \\
\hline Duration of MAX-WAG > 2 ppm (min), median (Q1, Q3) & $19.0(11.0,23.1)$ & $13.5(3.5,21.0)$ & 0.27 \\
\hline Proportion of MAX-WAG > 2 ppm during collection (\%), median (Q1, Q3) & $29.7 \%(19.8,47.2)$ & $21.2 \%(6.5,39.8)$ & 0.33 \\
\hline
\end{tabular}


Table 3. Maximum waste anesthetic gas within the nurse's breathing zone

\begin{tabular}{|c|c|c|c|}
\hline \multirow[b]{2}{*}{ Variable } & \multicolumn{2}{|c|}{ Randomization group } & \multirow[b]{2}{*}{$P$ value } \\
\hline & Traditional mask (control) & ISO-Gard mask & \\
\hline All patients (n) & 56 & 52 & \\
\hline Collection duration (min), mean \pm SD & $60.6 \pm 20.6$ & $56.9 \pm 9.7$ & $0.24 *$ \\
\hline Duration of MAX-WAG > $2 \mathrm{ppm}$ (min), median (Q1, Q3) & $3.0(1.5,6.5)$ & $1.0(0,3.0)$ & $<0.01$ \\
\hline Proportion of MAX-WAG >2 ppm during collection (\%), median (Q1, Q3) & $4.7 \%(2.4,9.8)$ & $2.0 \%(0,5.7)$ & $<0.01$ \\
\hline Anesthesia with desflurane (n) & 32 & 31 & \\
\hline Collection duration (min), mean \pm SD & $64.0 \pm 25.9$ & $58.1 \pm 9.1$ & $0.24 *$ \\
\hline Duration of MAX-WAG > $2 \mathrm{ppm}$ (min), median (Q1, Q3) & $3.8(1.5,9.5)$ & $1.0(0,3.0)$ & $<0.01$ \\
\hline Proportion of MAX-WAG >2 ppm during collection (\%), median (Q1, Q3) & $5.9 \%(2.6,15.0)$ & $1.5 \%(0,5.7)$ & $<0.01$ \\
\hline Anesthesia with sevoflurane (n) & 24 & 21 & \\
\hline Collection duration (min), mean \pm SD & $56.0 \pm 8.7$ & $55.1 \pm 10.6$ & $0.76^{*}$ \\
\hline Duration of MAX-WAG > 2 ppm (min), median (Q1, Q3) & $2.0(1.0,5.4)$ & $1.5(0.5,3.0)$ & 0.21 \\
\hline Proportion of MAX-WAG > 2 ppm during collection (\%), median (Q1, Q3) & $4.0 \%(1.7,8.0)$ & $2.6 \%(1.0,5.8)$ & 0.24 \\
\hline
\end{tabular}

\section{DISCUSSION}

Patient care during surgery and in the PACU requires medical staff to perform to their best professional capacity. Though it is known that work efficiency may decrease as a result of fatigue, scientific evidence has demonstrated that breathing WAG contributes to fatigue and work impairment. ${ }^{7}$ In addition, animal studies have shown that exposure to high levels of nitrous oxide and halogenated gases can cause cellular, mutagenic, carcinogenic, and teratogenic effects. Exposed groups have a reported higher incidence of infertility, spontaneous abortion, congenital abnormalities, premature births, cancer, and renal and hepatic disease. ${ }^{8}$

The operating room has been classically linked to exposure to high concentrations of WAG, but little is known about potential hazards associated with continuous trace exposure in the PACU. 'Traditionally, the PACU is not considered an area with increased risk of exposure to WAG, which is why scavenging devices are not routinely used. However, recent studies indicate that the possibility of exceeding NIOSH-recommended guidelines in the PACU exists.

This study was based on a previous pilot study on the exposure of nurses in the PACU to WAG, which found that PACU nurses were potentially exposed to higher than recommended levels of WAG $(<2 \mathrm{ppm}){ }^{6}$ Similarly, McGlothin et al found significantly higher than recommended concentrations of WAG within the patient's breathing zone during the first 15 minutes of the PACU stay. ${ }^{10}$ They also found that PACU nurses were exposed to significantly higher than recommended concentrations of WAG within their breathing zone and within 6 feet of patients, where concentrations of nitrous oxide were up to three times higher than that of controls who were using scavenging devices. ${ }^{10}$

Upon PACU arrival, measurable amounts of WAG will still be exhaled by patients because ventilation is the primary method of elimination. During this time, the most intensive bedside nursing is required, thus constituting an underestimated period of occupational risk for PACU nurses. ${ }^{11}$ Another study concluded that peak WAG levels were detected 1 hour after patient arrival to the PACU, contrary to the initial hypothesis in which investigators expected peak WAG levels to occur at admission. ${ }^{12}$ The location where the airway device is removed should also be considered; patients whose device is removed in the PACU versus the operating room yield higher initial concentrations of WAG than those who arrive to the PACU without a device. ${ }^{13}$ In our study, all patients were extubated prior to PACU arrival.

To address this issue, our study aimed to verify whether NIOSH guidelines regarding WAG exposure were being met and assess a possible solution. Our data suggest that patients using the ISO-Gard mask had a statistically significant shorter duration and lower median proportion of exposure to MAX-WAG $(P=0.02$ and 0.03 , respectively). The nurses also had a similar outcome $(P<0.01$ for both) (Figure 2$)$. This indicates the efficacy of the ISO-Gard mask in scavenging WAG and reducing exposure to patients and nurses in the PACU.

Subgroup analysis of patient and nurse breathing zones suggested a statistically significant difference in the duration and median proportion of MAX-WAG ( $>2 \mathrm{ppm}$ ) between the two groups and the desflurane subgroup. No significant differences were noted in the group that received sevoflurane. 


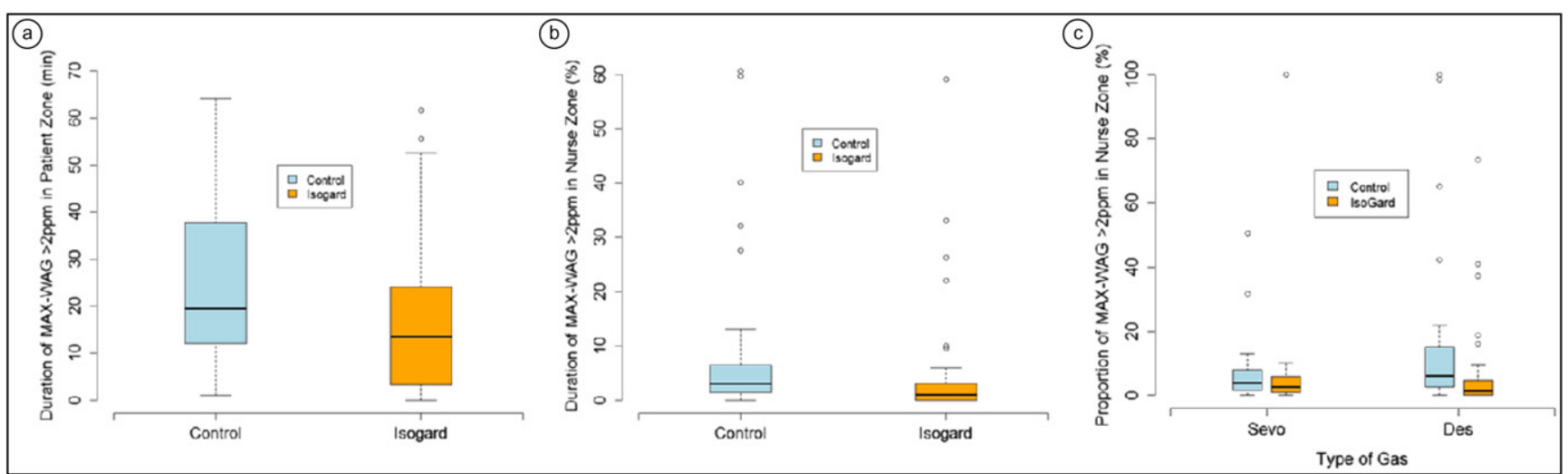

Figure 2. (a) Duration of maximum waste anesthetic gas (MAX-WAG) $>2$ ppm in patient's breathing zone. (b) Duration of MAX-WAG $>2$ ppm in nurse's breathing zone. (c) Proportion of MAX-WAG $>2$ ppm in nurse's breathing zone.

As a side note, the sample size of patients who received sevoflurane was smaller than that for desflurane (43/108 vs 65/108), which may have influenced this finding.

Though it is clear that elevated levels of WAG occur in the PACU nurse breathing zone, our study was not designed to obtain the long-term outcomes of participating nurses. As such, the precise consequences of long-term exposure to trace WAG levels in the PACU setting remain undetermined. Additionally, we did not measure the cumulative doses of WAG for each PACU nurse; the investigators considered doing so, but available measuring technology was expected to have resulted in inaccurate readings.

Another limitation is that precise dosing of volatile anesthetics was not recorded. Dosing was practitioner dependent, and more than 40 different anesthesiologists administered volatile anesthetics to study patients. In general, overall exhaled levels of sevoflurane were lower than those of desflurane, though this would be anticipated given the higher concentration of desflurane needed to achieve appropriate minimum alveolar concentration levels. Hiller et al provide further discussion on the pharmacokinetics of volatile anesthetics. $^{11}$

Studies have demonstrated that to maximize effectiveness in controlling WAG exposure, practices such as appropriate mask size selection and adjustment, minimal talking, mouth breathing by the patient, and use of a vacuum scavenging system must be implemented. ${ }^{14}$ We strongly encourage investigators and government agencies to work in conjunction to update existing protocols related to occupational WAG exposure and include the PACU setting as a potential hazard location in which standard scavenging measurements should be practiced.

In conclusion, patients continue to expel WAG at concentrations $>2 \mathrm{ppm}$ during the 1-hour postoperative period, with PACU nurses intermittently being exposed to WAG at concentrations $>2 \mathrm{ppm}$ during this time frame. The ISOGard mask effectively reduces the amount of WAG detected in the immediate 1-hour postoperative period in both the patient and nurse breathing zones. The long-term consequence of this exposure to PACU nursing staff remains undetermined.

\section{ACKNOWLEDGMENTS}

The authors thank the PACU nursing team at Memorial Hermann Hospital-Texas Medical Center, whose cooperation and participation made this project possible.

\section{FUNDING}

The authors acknowledge work attributed to and financial support provided by Teleflex, Incorporated (Raleigh, NC), and The University of Texas Medical School at Houston, Department of Anesthesiology. Dr. Cai's research was supported by the National Institutes of Health's Clinical and Translational Science Award Grant (UL1 TR000371).

\section{ORCID}

Tariq A. Syed (D) http://orcid.org/0000-0001-7351-5572

Tyrone Burnett (D) http://orcid.org/0000-0002-1169-770X

Stephanie H. Ahn (D) http://orcid.org/0000-0002-8280-6707

Chunyan Cai (D) http://orcid.org/0000-0002-7213-621X

1. US Department of Labor. Anesthetic gases: guidelines for workplace exposures. https://www.osha.gov/dts/osta/anestheticgases/. Updated May 18, 2000. Accessed June 27, 2016.

2. Smith FD. Management of exposure to waste anesthetic gases. AORN J. 2010;91:482-494.

3. National Institute for Occupational Safety and Health. Waste Anesthetic Gases: Occupational Hazards in Hospitals [NIOSH Pub. No. 2007-151]. Washington, DC: NIOSH; 2007. https://www.cdc.gov/ niosh/docs/2007-151/pdfs/2007-151.pdf.

4. Division of Occupational Health and Safety WAG Program Manager. Waste Anesthetic Gas. Washington, DC: National Institutes of Health Office of Research Services; 2018. http://www.ors.od.nih.gov/sr/dohs/ Documents/Waste\%20Anesthetic\%20Gas\%20(WAG)\%20Surveillan ce\%20Program.pdf.

5. Sessler DI, Badgwell JM. Exposure of postoperative nurses to exhaled anesthetic gases. Anesth Analg. 1998;87:1083-1088. doi: 10.1097/ 00000539-199811000-00019.

6. Williams G, Ramirez-Chapman A, Miller C. Exposure of nurses in the PACU to waste anesthetic gas-a pilot study. Int J Anesthesiol. 2014;33:1-4. https://print.ispub.com/api/0/ispub-article/19060. 
7. Smith G, Shirley AW. A review of the effects of trace concentrations of anesthestic on performance. Br J Anaesth. 1978;50:701-712. doi: 10.1093/bja/50.7.701.

8. Rowland AS, Baird DD, Weinberg CR, et al. Reduced fertility among women employed as dental assistants exposed to high levels of nitrous oxide. $N$ Engl J Med. 1992;327:993-997. doi: 10.1056/ NEJM199210013271405.

9. McGlothin JD, Moenning JE. Waste anesthesia gases in the healthcare environment: risks and prevention strategies. Clinical Foundations. 2013; http://www.clinicalfoundations.org/waste-anesthesia.html. Accessed June 27, 2016.

10. McGlothlin JD, Moenning JE, Cole SS. Evaluation and control of waste anesthetic gases in the postanesthesia care unit. J Perianesth Nurs. 2014;29:298-312. doi:10.1016/ j.jopan.2013.09.010.
11. Hiller KN, Altamirano AV, Cai C, Tran SF, Williams GW. Evaluation of waste anesthetic gas in the postanesthesia care unit within the patient breathing zone. Anesthesiol Res Pract. 2015;2015:1. doi: $10.1155 / 2015 / 354184$.

12. Krenzischek DA, Schaefer J, Nolan M, et al. Phase I collaborative pilot study: Waste anesthetic gas levels in the PACU. J Perianesth Nurs. 2002;17:227-239. doi: 10.1053/jpan.2002.34166.

13. Cheung S, Ozelsel T, Rashiq S, Tsui BC. Postoperative environmental anesthetic vapour concentrations following removal of the airway device in the operating room versus the postanesthesia care unit. Can J Anaesth. 2016;63:1016-1021. doi: 10.1007/s12630-016-0678-y.

14. Rademaker AM, McGlothlin JD, Moenning JE, et al. Evaluation of two nitrous oxide scavenging system using infrared thermography to visualize and control emissions. J Am Dent Assoc. 2009;140:190-199. doi: $10.14219 /$ jada.archive.2009.0133. 